10

\title{
Акустические векторные солитоны в анизотропных средах
}

\section{(ㄷ Г.Т. Адамашвили, М.Д. Пейкришвили, Р.Р. Коплатадзе}

Грузинский технический университет, Тбилиси, Грузия

E-mail: guram_adamashvili@ymail.com

\section{Поступило в Редакцию 23 июня 2016 г.}

Построена теория акустических векторных солитонов самоиндуцированной прозрачности в анизотропных средах. Показано, что в этих системах может формироваться двухкомпонентный векторный солитон, осциллирующий на суммарной и разностной частотах в окрестности частоты несущей акустической волны. Приводятся явные аналитические выражения для формы и параметров нелинейной волны, которые зависят от направления распространения импульса.

DOI: 10.21883/PJTF.2017.07.44464.16383

Распространение акустических двухкомпонентных векторных солитонов является одним из наиболее важных нелинейных эффектов в акустических системах. В зависимости от механизмов формирования акустических нелинейных волн отличают резонансные и нерезонансные нелинейные волны. Резонансные акустические нелинейные волны стационарной формы (солитоны, бризеры, векторные солитоны) могут формироваться в условиях нелинейного когерентного взаимодействия акустического импульса с содержащимися в среде парамагнитными примесями или квантовыми точками, когда выполняются условия акустической самоиндуцированной прозрачности (АСИП): $T \ll T_{1,2}$, $\omega T \gg 1$, где $T$ и $\omega$ - длительность и частота несущей волны импульса, $T_{1}$ и $T_{2}$ - времена продольной и поперечной релаксаций парамагнитных примесей или квантовых точек [1]. При этом, когда площадь огибающей тензора деформации акустического импульса $\Theta$ превышает $\pi$, образуется солитон (2л-импульс) [2], а когда выполняется условие $|\Theta| \ll 1$, может формироваться бризер (0л-импульс) [3]. Однако бризеры не являются единственно возможными резонансными нелинейными волнами малой энергии (амплитуды). В работах $[4,5]$ было показано, что в условиях АСИП, наряду с однокомпонентными 
бризерами, резонансными нелинейными волнами малой амплитуды могут быть также и двухкомпонентные векторные солитоны АСИП. Эти нелинейные волны могут осциллировать на суммарной и разностной частотах, в окрестности частоты несущей акустической волны. Такие двухкомпонентные векторные солитоны АСИП, так же как и однокомпонентные бризеры, являются 0л-импульсами АСИП.

В изотропных средах свойства этих нелинейных акустических волн изучены достаточно подробно (см., например, [2] и цитированные там работы). В анизотропных средах ситуация существенно меняется. В частности, в таких средах могут распространяться необыкновенные акустические волны, для которых, в отличие от изотропных сред, вектор групповой скорости акустической волны не коллинеарен волновому вектору [6-8]. Явления АСИП в анизотропных средах для солитонов исследованы в работе [9]. Однако ситуация существенно меняется, когда в анизотропных средах распространяются акустические двухкомпонентные векторные солитоны АСИП. Цель настоящей работы рассмотреть вопрос о формировании акустических двухкомпонентных векторных солитонов АСИП в анизотропных средах.

В качестве модели, позволяющей исследовать акустические двухкомпонентные векторные солитоны АСИП в анизотропных средах, рассмотрим одноосный кристалл гексагональной системы, содержащий малую концентрацию $n_{0}$ парамагнитных примесей или квантовых точек с эффективным электронным спином $S=1 / 2$. Предположим, что линейно поляризованный вдоль оси $x$ акустический импульс плоской волны с длительностью $T$, частотой $\omega \gg T^{-1}$, волновым вектором $\mathbf{k}$ распространяется вдоль оси $\xi$, которая составляет угол $\theta$ с осью $z$. В силу изотропии среды в плоскости $x y$, без ограничения общности можно предположить, что волновой вектор лежит в плоскости $y z$. Предположим, что внешнее постоянное магнитное поле $H_{0}$ направлено вдоль оси шестого порядка, которая совпадает с координатной осью $z$.

$\varepsilon_{x z}$-компонента тензора деформации необыкновенной акустической волны удовлетворяет волновому уравнению движения $[6,9]$

$$
\frac{\partial^{2} \varepsilon_{x z}}{\partial t^{2}}=C(\theta) \frac{\partial^{2} \varepsilon_{x z}}{\partial \xi^{2}}+\cos ^{2} \theta \frac{L}{\rho} \frac{\partial^{2}\left\langle S_{x}\right\rangle}{\partial \xi^{2}},
$$

где $\rho$ - плотность среды, $\varepsilon_{x z}=\frac{1}{2} \frac{\partial u_{x}}{\partial z}=\frac{1}{2}\left(\varepsilon^{+}+\varepsilon^{-}\right), u_{x}-$ компонента вектора деформации $\bar{u}\left(u_{x}, u_{y}, u_{z}\right)$ акустического поля импульса, 
$L=\beta_{0} H_{0} F_{x z x z}, \beta_{0}$ - магнетон Бора, $F_{x z x z}$ - компонента тензора спинфононной связи. Зависимость $\left\langle S_{x}\right\rangle$ от тензора деформации определяется из магнитных уравнений Блоха [10]. В акустически одноосной среде в гексагональном кристалле, имеют место следующие соотношения: $\lambda_{x y x y}=b, \lambda_{x z x z}=\lambda_{y z y z}=d$, где под компонентами $\lambda_{x k l m}$ подразумеваем адиабатические значения модулей упругости

$$
C(\theta)=\frac{1}{\rho}\left(b \sin ^{2} \theta+d \cos ^{2} \theta\right) .
$$

Для плоской волны имеет место трансляционная инвариантность в направлении вдоль оси $x$ и, следовательно, все функции не зависят от $x$, т. е. $\frac{\partial}{\partial x}=0$. При этом $\xi=z \cos \theta+y \sin \theta$.

Для решения уравнения (1) в форме векторного солитона АСИП в анизотропных средах перейдем к медленным переменным с помощью выражений

$$
\varepsilon_{x z}=\frac{1}{2} \sum_{l= \pm 1} \widehat{E}_{l} Z_{l}, \quad\left\langle S_{x}\right\rangle=\frac{i}{2} \sum_{l= \pm 1} l \rho^{l} Z_{-l}
$$

где $Z_{l}=\exp [i l(k \xi-\omega t)], \widehat{E}_{l}$ и $\rho^{l}-$ медленно меняющиеся комплексные амплитуды $\varepsilon_{x z}$-компоненты тензора деформации и намагниченности соответственно.

Подставляя выражения (2) в уравнение (1), получим уравнения АСИП в медленных переменных

$$
\begin{gathered}
\frac{\partial \rho^{l}}{\partial t}=i l \Delta \rho^{l}-\frac{L}{\hbar}\left\langle S_{z}\right\rangle \widehat{E}_{-l}, \\
\frac{\partial\left\langle S_{z}\right\rangle}{\partial t}=\frac{L}{2 \hbar} \sum_{l= \pm 1} \rho^{l} \widehat{E}_{l}, \\
\sum_{l= \pm 1} Z_{l}\left(-2 i l \omega \frac{\partial \hat{E}_{l}}{\partial t}-2 i l k C^{2} \frac{\partial \hat{E}_{l}}{\partial \xi}+\frac{\partial^{2} \widehat{E}_{l}}{\partial t^{2}}-C^{2} \frac{\partial^{2} \widehat{E}_{l}}{\partial \xi^{2}}\right. \\
\left.-l \cos ^{2} \theta \frac{L}{\rho} i k^{2} \rho^{-l}\right)=0
\end{gathered}
$$

и закон дисперсии для необыкновенной акустической волны $\omega^{2}=C^{2} k^{2}$. Здесь $\hbar-$ постоянная Планка, $\Delta=\omega_{0}-\omega, \omega_{0}$ - частота спинов.

2* Письма в ЖТФ, 2017, том 43, вып. 7 
Уравнения системы (3) являются уравнениями АСИП в одноосных кристаллах и могут описать широкий спектр нелинейных явлений.

Для анализа этих уравнений воспользуемся пертурбативным методом редукции $[4,11]$, согласно которому функцию $\Theta_{l}=\int_{-\infty}^{t} \widehat{E}_{l} d t$ при условии $\left|\Theta_{l}\right| \ll 1$ можно представить в форме

$$
\Theta_{l}(\xi, t)=\sum_{\alpha=1}^{\infty} \varepsilon^{\alpha} \Theta_{l}^{(\alpha)}=\sum_{\alpha=1}^{\infty} \sum_{n=-\infty}^{\infty} \varepsilon^{\alpha} Y_{l, n} f_{l, n}^{(\alpha)}(\eta, \tau),
$$

где $\varepsilon$ - малый параметр,

$$
\begin{gathered}
Y_{l, n}=\exp \left[i n\left(Q_{l, n} \xi-\Omega_{l, n} t\right)\right], \quad \eta_{l, n}=\varepsilon Q_{l, n}\left(\xi-v_{g(l, n)} t\right), \\
\tau=\varepsilon^{2} t, \quad v_{g(l, n)}=\frac{d \Omega_{l, n}}{d Q_{l, n}} .
\end{gathered}
$$

Такое представление позволяет выделить из $\Theta_{l}$ еще более медленно меняющиеся функции $f_{l, n}^{(\alpha)}$. Следовательно, предполагается, что величины $\Omega, Q$ и $f_{l, n}^{(\alpha)}$ удовлетворяют неравенствам

$$
\omega \gg \Omega, \quad k \gg Q, \quad\left|\frac{\partial f_{l, n}^{(\alpha)}}{\partial t}\right| \ll \Omega f_{l, n}^{(\alpha)}, \quad\left|\frac{\partial f_{l, n}^{(\alpha)}}{\partial z}\right| \ll Q f_{l, n}^{(\alpha)} .
$$

Величины $Q$ и $\Omega$ зависят от $l$ и $n$, но в дальнейшем, для простоты, индексы $l$ и $n$ опустим там, где это не приведет к путанице.

Подставляя разложение (4) в систему уравнений (3), получаем нелинейное уравнение

$$
\begin{gathered}
\sum_{\alpha=1}^{\infty} \sum_{l= \pm 1} \sum_{n=-\infty}^{\infty} \varepsilon^{\alpha} Y_{l, n} Z_{l}\left(\tilde{W}_{l, n}+\varepsilon J_{l, n} \frac{\partial}{\partial \eta}+\varepsilon^{2} h_{l, n} \frac{\partial}{\partial \tau}+i \varepsilon^{2} H_{l, n} \frac{\partial^{2}}{\partial \eta^{2}}\right) f_{l, n}^{(\alpha)} \\
=-i \varepsilon^{3} \beta_{0}^{2} \sum_{l= \pm 1} l Z_{l} \int \frac{\partial \Theta_{l}^{(1)}}{\partial t} \Theta_{-l}^{(1)} \Theta_{l}^{(1)} d t^{\prime}+O\left(\varepsilon^{4}\right)
\end{gathered}
$$

где

$$
\tilde{W}_{l, n}=i n \Omega\left[A_{l} n \Omega-B_{l} n Q+n^{2} \Omega^{2}-C^{2} n^{2} Q^{2}-\frac{l}{n} \frac{K}{\Omega}\right],
$$




$$
\begin{gathered}
J_{l, n}=n Q\left[2 A_{l} \Omega v_{g}-B_{l}\left(Q v_{g}+\Omega\right)+3 n v_{g} \Omega^{2}-C^{2} n Q\left(Q v_{g}+2 \Omega\right)\right] \\
h_{l, n}=-2 A_{l} n \Omega+B_{l} n Q-3 n^{2} \Omega^{2}+C^{2} n^{2} Q^{2} \\
H_{l, n}=Q^{2}\left[-A_{l} v_{g}^{2}+B_{l} v_{g}-3 n \Omega v_{g}^{2}+C^{2} n\left(2 Q v_{g}+\Omega\right)\right] \\
K(\theta)=\cos ^{2} \theta \frac{L^{2} n_{0} k^{2}}{2 \rho \hbar} \int \frac{g(\Delta) d \Delta}{1+T^{2} \Delta^{2}} \\
\beta_{0}^{2}(\theta)=\cos ^{2} \theta \frac{L^{4} n_{0} k^{2}}{4 \rho \hbar^{3}} \int \frac{g(\Delta) d \Delta}{1+T^{2} \Delta^{2}} \\
A_{l}=2 l \omega, \quad B_{l}=2 l k C^{2}
\end{gathered}
$$

$g(\Delta)$ - нормированная функция неоднородного уширения спектральной линии.

Из уравнения (5) следует, что из всех величин $f_{l, n}^{(\alpha)}$ отличны от нуля только члены $f_{+1, \pm 1}^{(1)}$. Для определения этих функций приравняем нулю по отдельности члены при одинаковых степенях $\varepsilon$. В результате получим связь между величинами $Q$ и $\Omega$

$$
A_{l} n \Omega-B_{l} n Q+n^{2} \Omega^{2}-C^{2} n^{2} Q^{2}-\frac{l}{n} \frac{K}{\Omega}=0
$$

и связанные нелинейные уравнения Шредингера для функции $u_{ \pm}=$ $=\varepsilon f_{+1, \pm 1}^{(1)}$

$$
-i\left(\frac{\partial u_{ \pm}}{\partial t}+v_{ \pm} \frac{\partial u_{ \pm}}{\partial z}\right)+p_{ \pm} \frac{\partial^{2} u_{ \pm}}{\partial z^{2}}+g_{ \pm}\left|u_{ \pm}\right|^{2} u_{ \pm}+r_{ \pm}\left|u_{ \pm}\right|^{2} u_{ \pm}=0,
$$

где

$$
v_{ \pm}=v_{g(+1, \pm 1)}, \quad p_{ \pm}=\frac{H_{+1, \pm 1}}{Q^{2} h_{+1, \pm 1}}, \quad g_{ \pm}=\frac{\beta_{0}^{2}}{h_{+1, \pm 1}}, \quad r_{ \pm}=g_{ \pm}\left(1-\frac{\Omega_{ \pm}}{\Omega_{ \pm}}\right) .
$$

Эти уравнения имеют двухкомпонентное солитонное решение в следующей форме [4]:

$$
u_{ \pm}(\xi, t)=\frac{f_{ \pm 1}}{b T} \operatorname{sech}\left(\frac{t-\frac{\xi}{V_{0}}}{T}\right) \exp \left[i\left(k_{ \pm 1} \xi-\omega_{ \pm 1} t\right)\right]
$$

Письма в ЖТФ, 2017, том 43, вып. 7 
где $V_{0}-$ скорость нелинейной волны. При этом выполняются неравенства $k_{ \pm 1} \ll Q_{ \pm 1}, \omega_{ \pm 1} \ll \Omega_{ \pm 1} ; f_{ \pm 1}, k_{ \pm 1}$ и $\omega_{ \pm 1}$ являются вещественными постоянными, которые определяются из уравнений

$$
\begin{gathered}
f_{+1}^{2}=\frac{p_{+} g_{-}-p_{-} r_{+}}{p_{-} g_{+}-p_{+} r_{-}} f_{-1}^{2}, \quad k_{ \pm}=\frac{V_{0}-v_{ \pm}}{2 p_{ \pm}}, \\
\omega_{+1}=\frac{p_{+}}{p_{-}} \omega_{-1}+\frac{V_{0}^{2}\left(p_{-}^{2}-p_{+}^{2}\right)+v_{-}^{2} p_{+}^{2}-v_{+}^{2} p_{+}^{2}}{4 p_{+} p^{2}} .
\end{gathered}
$$

Подставляя солитонные решения (9) для функции $u_{ \pm}$в уравнения (8), получаем для компоненты тензора деформации выражение

$$
\begin{aligned}
\varepsilon_{x z}= & \frac{1}{b T} \operatorname{sech}\left(\frac{t-\frac{\xi}{V_{0}}}{T}\right)\left\{( \Omega _ { + } + \omega _ { + 1 } ) f _ { 1 } \operatorname { s i n } \left[\left(k+Q_{+}+k_{+1}\right) z\right.\right. \\
& \left.-\left(\omega+\Omega_{+}+\omega_{+1}\right) t\right]-\left(\Omega_{-}-\omega_{-1}\right) f_{2} \sin \left[\left(k-Q_{-}+k_{-1}\right) z\right. \\
& \left.\left.-\left(\omega-\Omega+\omega_{-1}\right) t\right]\right\},
\end{aligned}
$$

где

$$
b^{2}=V_{0}^{2} \frac{f_{+1}^{2} g_{+}+f_{-1}^{2} r_{+}}{2 p_{+}}, \quad T^{-2}=V_{0}^{2} \frac{v_{+} k_{+1}+k_{+1}^{2} p_{+}-\omega_{+1}}{p_{+}} .
$$

Появление в выражении (11) тригонометрических функций указывает на возникновение периодических осцилляций по времени и координате с характерными параметрами на суммарной $\omega+\Omega+$ $+\omega_{+1}\left(k+Q_{+}+k_{+1}\right)$ и разностной $\omega-\Omega_{-}+\omega_{-1}\left(k-Q_{-}+k_{-1}\right)$ частотах (волновых чисел) в окрестности частоты $\omega$ (волнового числа $k$ ) несущей волны, в результате чего солитонные решения (9) для величин $u_{ \pm}$трансформируются в решение (11) для величины $\varepsilon_{x z}$, имеющей вид двухкомпонентного векторного солитона. Параметры нелинейной волны (11) определяются из выражений $(6),(10)$ и (12). Связь между величинами $\Omega_{ \pm}$и $Q_{ \pm}$определяется из уравнения (7).

Из закона дисперсии $\omega^{2}=C^{2} k^{2}$ можно определить направление групповой скорости необыкновенной волны $\operatorname{tg} \vartheta=\operatorname{tg} \theta \frac{\lambda_{x y y x}}{\lambda_{x z z x}}$, где $\vartheta-$ угол между осью $z$ и направлением групповой скорости.

Из уравнений волны (6), (10) и (12) очевидно, что, в отличие от изотропных сред, характеризующие параметры необыкновенной 
нелинейной волны $\varepsilon_{ \pm}, C, K, \beta_{0}^{2}, f_{ \pm 1} \omega_{ \pm 1} k_{ \pm 1}, v_{ \pm}, \Omega_{ \pm}$и $Q_{ \pm}$зависят от угла $\theta$, т.е. от направления распространения импульса необыкновенной волны.

Представленные аналитические результаты позволяют надеяться на то, что исследованные двухкомпонентные солитоны АСИП необыкновенной волны можно наблюдать экспериментально, так как параметры векторного солитона того же порядка, что и в изотропных средах.

Работа выполнена в рамках проекта № 217064 ННФ им. Ш. Руставели.

\section{Список литературы}

[1] Shiren N. // Phys. Rev. 1970. V. 2. P. 2471.

[2] Adamashvili G.T., Kaup D.J. // Phys. Lett. A. 2012. V. 376. P. 432-437.

[3] Адамашвили Г.Т., Адамашвили Н.Т., Моцонелидзе Г.Н., Пейкришвили М.Д. // Письма в ЖТФ. 2006. Т. 32. С. 82.

[4] Adamashvili G.T. // Phys. Rev. E. 2016. V. 93. P. 023002.

[5] Adamashvili G.T. // Phys. Rev. E. 2012. V. 85. P. 067601.

[6] Ландау Л.Д., Лифиии Е.М. Теория упругости. М.: Наука, 1987.

[7] Abo-el-nour Abd-alla, Askar N.A. // Appl. Math. Inf. Sci. 2014. V. 8. P. 1625.

[8] Alshits V.I., Bessonov D.A., Lyubimov V.N. // Crystallogr. Reports. 2013. V. 58. P. 867.

[9] Адамашвили Г.Т. // ФТТ. 1983. Т. 25. С. 1872.

[10] Альтшулер С.А., Козырев Б.М. Электронный парамагнитный резонанс соединений элементов промежуточных групп. М.: Наука,1972.

[11] Taniuti T., Iajima N. // J. Math. Phys. 1973. V. 14. P. 1389. 